Review article

\title{
Parkin-linked Parkinson's disease: From clinical insights to pathogenic mechanisms and novel therapeutic approaches
}

\author{
Kobi Wasner ${ }^{\mathrm{a}}$, Anne Grünewald ${ }^{\mathrm{a}, \mathrm{b}}$, Christine Klein ${ }^{\mathrm{b}, *}$ \\ a Luxembourg Centre for Systems Biomedicine, University of Luxembourg, Esch-sur-Alzette, Luxembourg \\ ${ }^{\mathrm{b}}$ Institute of Neurogenetics, University of Lübeck, Lübeck, Germany
}

\section{A R T I C L E I N F O}

\section{Article history:}

Received 1 August 2020

Accepted 4 August 2020

Available online $\mathrm{xxx}$

\section{Keywords:}

Parkin

Parkinson's disease

Mitochondria

Inflammation

Gene-specific therapy

Clinical trial

\begin{abstract}
A B S T R A C T
With over 7 million patients worldwide, Parkinson's disease (PD) is becoming more prevalent as life span and industrialization increase. While the majority of cases are sporadic and present in individuals over 65, inherited mutations in Parkin can manifest in individuals as young as teenagers. The involvement of Parkin in neurodegeneration has been widely investigated and its role in mitophagy is undeniable. In the recent years, however, additional functions of the protein are beginning to come to light, which in turn may influence the way patients harboring Parkin mutations are treated. In the present article, we discuss the clinical and genetic aspects of Parkin-linked PD. For this purpose, we consulted the MDSGene database, which comprises the literature of more than 1000 patients with Parkin mutations. In addition, we provide insight into Parkin's multifaceted role in mitochondrial clearance and maintenance. Finally, we discuss treatment strategies such as brain stimulation, small molecule drugs and dopaminergic cell replacement that could be tailored to improve the clinical phenotypes in Parkin-linked PD.
\end{abstract}

(c) 2020 The Authors. Published by Elsevier B.V. This is an open access article under the CC BY-NC-ND license (http://creativecommons.org/licenses/by-nc-nd/4.0/).

\section{Contents}

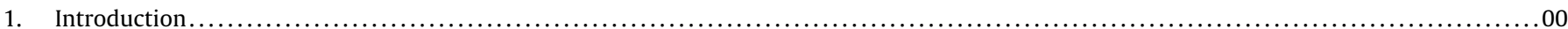

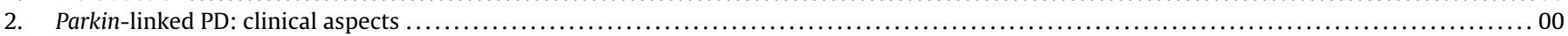

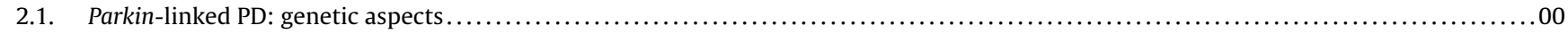

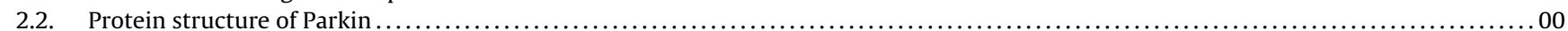

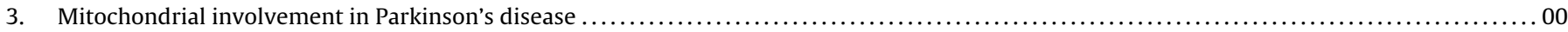

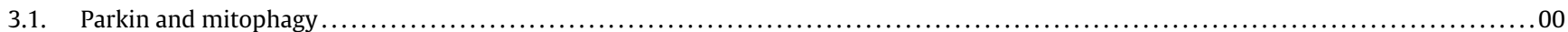

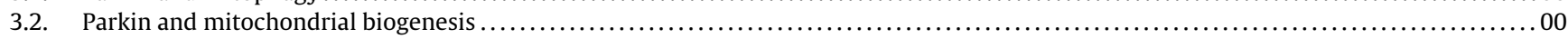

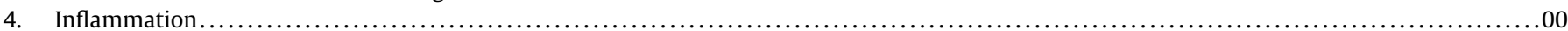

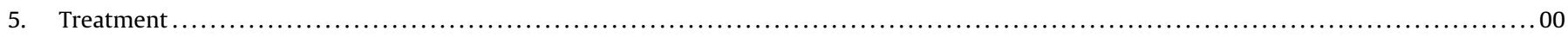

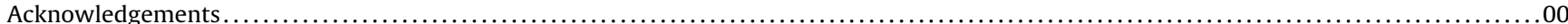

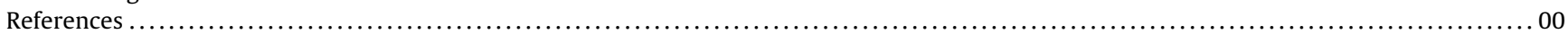

\section{Introduction}

While Parkinson's disease (PD) was first systematically described by the British physician James Parkinson in 1817, it took almost two centuries until the first recessively inherited form of PD was discovered by Kitada and colleagues in Japan in 1998 (Kitada et al., 1998). In his work entitled "An Essay on the Shaking Palsy",

\footnotetext{
* Corresponding author: Institute of Neurogenetics, University of Lübeck, MariaGoeppert-Str. 1, 23562 Lübeck, Germany.

E-mail addresses: kobi.wasner@uni.lu (K.Wasner), anne.gruenewald@uni.lu (A. Grünewald), christine.klein@neuro.uni-luebeck.de (C. Klein).
}

James Parkinson characterized the symptoms of six individuals including some patients and individuals on the street - with what he named paralysis agitans - a malady resulting in, "involuntary tremulous motion, with lessened muscular power, in parts not in action and even when supported" (Parkinson, 2002). This condition was later named after James Parkinson and, in reference to the term 'Parkinson's disease', Kitada et al. chose to name their newly identified gene 'Parkin'. Although the most striking clinical difference between Parkin mutation carriers and classical PD was the very early, juvenile age at onset (AAO) in the former, the clinical presentation caused by Parkin mutations was compatible with a diagnosis of PD, albeit characterized by a strong susceptibility to dopa-induced dyskinesia and motor fluctuations. Further

https://doi.org/10.1016/j.neures.2020.09.001

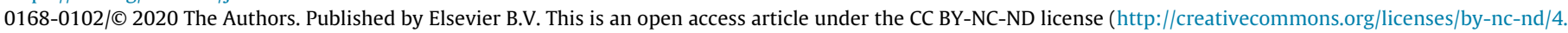
$0 /)$. 
extending the close phenotypic link of Parkin-linked PD and 'idiopathic' PD, i.e. PD of unknown origin and typically late AAO, Parkin mutations were soon identified also in a family with late-onset tremor-dominant PD (Klein et al., 2000).

The exact frequency of Parkin mutations is currently unknown, which is especially true for the most common, late-onset patient population where systematic mutational screens are lacking. However, Parkin mutations are overall rare and, even in the younger AAO groups ( $<50$ years) account for $\sim 2.6 \%$ of the cases only (Tan et al., 2019). Mutation frequency increases with decreasing AAO and is highest in the juvenile group (AAO < 20 years) where biallelic Parkin mutations are found in up to $77 \%$ of the patients (Lucking et al., 2000). Given the recent 'PD Pandemic' with PD being the fastest growing neurological disease with a current estimated global number of $\sim 7$ million PD patients (Dorsey and Bloem, 2018), one may expect a total of 35,000-70,000 Parkin cases worldwide when assuming an estimated fraction of Parkin mutation carriers across all AAO groups of $0.5-1 \%$.

\section{Parkin-linked PD: clinical aspects}

The Movement Disorder Society Genetic mutation database (MDSGene; www.mdsgene.org) provides a comprehensive online resource linking reported genetic mutations with movement disorder phenotypes as well as with demographic and other clinical information including PARK-Parkin (Kasten et al., 2018). MDSGene currently lists 1000 biallelic Parkin mutation carriers (44\% female) with PD and a median AAO of 31 years $\left(25^{\text {th }} / 75^{\text {th }}\right.$ percentile: $23 / 39$ years; range: 3-73 years). The most common presenting sign is tremor, followed by bradykinesia and dystonia. Indeed, across all listed patients, dystonia is the most common clinical feature after the cardinal PD signs and is present in $65 \%$ of those with available information. Underscoring the importance of dystonia as a prominent feature of Parkin-linked PD especially in the very early-onset patients, the percentage of patients with dystonia rises to $85 \%$ in those with a juvenile AAO, i.e. with an onset of PD at 20 years or younger. As already mentioned in the original description of the Parkin gene (Kitada et al., 1998), dyskinesia is a common finding in Parkin mutation carriers and found in $78 \%$ of all patients with available information on this feature and in even $90 \%$ of those with a juvenile AAO. Likewise, motor fluctuations occur at high frequency (87\%) and are only slightly more common in the juvenile onset group (91\%). Non-motor signs do occur in Parkin mutation carriers, however, reporting has been inconsistent and data missingness is high.

As information on the presence or absence of non-motor signs and symptoms has been provided for 100 Parkin mutation carriers (10\%) or fewer, $90 \%$ data missingness does not allow for meaningful conclusions on the frequency of the following features: autonomic signs or symptoms, sleep benefit, depression, anxiety, and psychotic signs and symptoms. By contrast, previous meta-analyses of published PD patients with Parkin mutations indicated that dementia is very rare, affecting less than $3 \%$ of cases (Grunewald et al., 2013; Kasten et al., 2018). The largest fraction of Parkin mutation carriers is of Asian origin (37\% overall; $44 \%$ in the juvenile AAO group), including $10 \%$ from Japan, followed by White European (35\%).

\subsection{Parkin-linked PD: genetic aspects}

To review the mutational spectrum of Parkin, we also consulted MDSGene and our in-house database on published heterozygous Parkin mutation carriers. To date, 200 different mutations have been published. These include 18 variations in introns, 13 nonsense mutations, 69 missense mutations, 29 small insertions or deletions, and 71 exon rearrangements (including 42 deletions and 26 multiplications). Out of 1155 mutation-positive index patients, 778 (67 \%) carried at least one exon rearrangement with exon 3 deletions being the most common and affecting $120(10 \%)$ index patients (on one or two mutant alleles).

When assessing the impact of different mutation types on the AAO, we did not observe any significant group differences across index patients with homozygous mutations (variants in introns: AAO [SD], 23.8 [10.1] years, $n=4$; nonsense mutations: 31.4 [8.1] years, $\mathrm{n}=8$; missense mutations: 35.0 [17.5] years, $\mathrm{n}=35$; small insertions/deletions: 31.0 [11.3], $\mathrm{n}=75$; exon rearrangements: 32.9 [11.5] years, $n=200$ ). The same observation was made when focusing exclusively on index patients with heterozygous mutations (variants in introns: n/a; nonsense mutations: AAO [SD], 30.2 [14.3] years, $n=5$; missense mutations: 42.3 [13.3] years, $n=125$; small insertions/deletions: 35.6 [12.3], $\mathrm{n}=27$; exon rearrangements: AAO [SD], 40.9 [13.8] years, $\mathrm{n}=238$ ).

By contrast, comparing the mean AAO of all index patients with biallelic (AAO [SD], 31.3 [12.0], $\mathrm{n}=663$ ) vs. those with heterozygous mutations (40.7 [13.7] years, $\mathrm{n}=409$ ), we determined a difference of about ten years ( $p<0.0001)$. With an AAO in the forties, Parkin heterozygotes suffer from $P D$ about ten years earlier than idiopathic patients who typically develop first signs in their $6^{\text {th }}$ decade of life (Grunewald et al., 2013). To obtain an even clearer picture of the influence of heterozygous Parkin mutations on the onset of PD, we repeated the aforementioned analysis for exon 3 deletion carriers only. Interestingly, in this homogenous (albeit small) subset, the AAO was as low in heterozygotes (AAO [SD], 33.1 [6.3], $\mathrm{n}=10$ ) as in homozygotes (32.6 [10.2], $\mathrm{n}=42$ ). Of note, for all AAO analyses, individuals with known digenic inheritance of PD-associated mutations were excluded.

\subsection{Protein structure of Parkin}

Parkin is a 465-residue protein involved in the ubiquitinproteasome system: a process which mediates the targeting of proteins for degradation. As an E3 ubiquitin ligase, Parkin recruits an E2 conjugating enzyme to facilitate the transfer of a ubiquitin substrate onto its target protein. The addition of ubiquitin onto a protein can produce a variety of effects including degradation via the proteasome, alteration of its cellular location, or promote or prevent protein-protein interactions (Seirafi et al., 2015).

Parkin belongs to the RING-between-RING family of E3 ligases, which generally comprises of two RING finger domains and an inbetween-RING region. RING1 serves as the binding site for an E2 ubiquitin conjugating enzyme, while RING2 contains an active site cysteine residue, which accepts and cleaves ubiquitin from the E2 enzyme and transfers it onto its substrates. A ubiquitin-like domain is also present at the $\mathrm{N}$-terminus of Parkin and plays a role in substrate recognition.

An ample amount of studies have found that Parkin is largely a cytosolic protein mediating ubiquitination in the cytosol. However, several studies have also shown small pools of Parkin localized to mitochondria (Kuroda et al., 2006; Narendra et al., 2008; Rothfuss et al., 2009).

\section{Mitochondrial involvement in Parkinson's disease}

The foundation of PD research was forever changed in the late $20^{\text {th }}$ century in California, United States. In the summer of 1982, emergency rooms in San Fransisco were abruptly met with peculiarly "frozen" patients: young women and men who were unable to move or speak, yet were conscious. Neurologist James William Langston recognized these symptoms as advanced PD and adminis- 
tered the only known effective treatment - L-DOPA - and "unfroze" the patients.

The patients were found to be drug users who ingested "China White" - a synthetic opioid analgesic produced by drug dealers. Analysis of the drug determined its chemical name: 1-metyl-4phenyl-1,2,3,6-tetrahydro pyridine (MPTP). Unbeknownst to them, this toxic compound was the perfect inhibitor of mitochondrial respiration.

\subsection{Parkin and mitophagy}

Damaged mitochondria are quickly degraded and replaced in the healthy brain. One of the most prominent and studied functions of Parkin is its role in mitophagy - the selective degradation of malfunctioning mitochondria by autophagy. Mitochondrial damage results in the depolarization of the outer mitochondrial membrane (OMM), thereby preventing PINK1 import through the OMM transmembrane protein complexes (Narendra et al., 2008). PINK1 subsequently accumulates along the OMM and begins to phosphorylate its targets, including ubiquitin and Parkin at serine 65 (Shiba-Fukushima et al., 2012). Phosphorylation at these sites activates and recruits Parkin to the OMM where Parkin subsequently begins to transfer ubiquitin onto its targets including mitofusins, VDACs and BAK (Fig. 1) (Bernardini et al., 2019; Geisler et al., 2010; Kane et al., 2014; Sarraf et al., 2013). Ubiquitin chain linkages on K48 primes degradation of the proteins by the proteasome, while K63 ubiquitination is recognized by autophagy adaptors, which eventually form the autophagasome, leading to its destruction by lysosomes.

Mitochondria are highly dynamic organelles, whereby a balance of degradation and biogenesis is mediated by morphological changes through fission and fusion (Deng et al., 2008). Mitofusin 1 and 2 (MFN1/2), which are downstream targets of Parkin, facilitate the fusion of the OMM, while Dynamin-related protein 1 (Drp1) regulates the fission of the inner mitochondrial membrane (IMM). Animal models lacking Parkin show striking mitochondria morphological phenotypes including swelling, herniation and broken cristae (Deng et al., 2008).

Mitophagy is a process performed by the cell in order to combat dysfunction and as a resort to prevent cell death. Once past a certain threshold and committing to a cell death fate, intrinsic apoptosis is mediated by the permeabilization of mitochondrial membranes and release of pro-apoptotic factors such as cytochrome C. Perforation of the mitochondrial membranes is executed by BAK and BAX, which are recruited to the OMM and form oligomers, which puncture the membranes (Fig. 1). Parkin reduces BAX localization to the OMM through an indirect interaction and also directly ubiquitinates BAK, preventing its development into oligomers and subsequent destruction of the OMM and release of cytochrome C (Bernardini et al., 2019). Thus, Parkin-mediated mitophagy also serves to prevent apoptosis.

During oxidative stress and mitophagy, mitochondria release signals to other organelles in response to a variety of stimuli. Mitochondrial-derived vesicles (MDVs) are filled with oxidized cargo that bud off mitochondria (independently of mitochondrial fission). Parkin colocalizes with MDVs and stimulates their formation in response to ROS (McLelland et al., 2014).

Recent evidence revealed Parkin's many functions other than governing mitophagy; Parkin ubiquitinates hundreds of targets, which play other roles in mitochondrial pathways, it contains the structural architecture common in several transcription factors and associates with the mitochondrial genome (Alves da Costa et al., 2018).

\subsection{Parkin and mitochondrial biogenesis}

Peroxisome proliferator-activated receptor gamma coactivator 1-alpha (PGC-1 $\alpha$ ) is a transcriptional coactivator known as the master regulator of mitochondrial biogenesis, where it transcribes a multitude of downstream target genes responsible for mitochondrial maintenance and respiration including nuclear respiratory factors (NRFs) and estrogen-related receptors (ERRs). Overexpression of PGC- $1 \alpha$ leads to an increase of these targets, mitochondrial-encoded proteins and mitochondrial DNA (mtDNA) copy number (Zheng et al., 2017).

One of Parkin's targets for ubiquitination, Parkin interacting substrate (PARIS), is a transcriptional repressor of PGC- $1 \alpha$ (Fig. 1). PARIS is ubiquitously expressed throughout the body and heterogeneously expressed throughout the brain, where it is localized to neurons, including midbrain dopaminergic neurons of the substantia nigra (SN) pars compacta. Consequently, PARIS protein accumulates in the brain of patients with autosomal recessive juvenile PD. Mutations to Parkin result in the accumulation of PARIS and successive inhibition of the transcription of PGC- $1 \alpha$ and its downstream targets (Shin et al., 2011; Zheng et al., 2017). Thus, Parkin is not only responsible for the degradation of damaged mitochondria, but also plays a role in the mitochondrial biogenesis pathway.

The generation of new mitochondria requires the replication and transcription of the mitochondrial genome, which are mainly regulated by nuclear-encoded proteins. PGC- $1 \alpha$ targets NRF1 and NRF2 - transcription factors, which activate the expression of molecules maintaining mtDNA (Gugneja et al., 1996). NRF-1 and NRF-2 transcribe TFAM and TFB2M, two molecules required for the initiation of transcription and replication of the mitochondrial genome.

NRF-1 abundance has been shown to be reduced in cells which lack Parkin (Shin et al., 2011; Stevens et al., 2015), and interestingly, NRF-1 was shown to contain binding sites in both Parkin and PINK1 promoter regions in silico, in vitro and in vivo (Suliman et al., 2017). This was supported by overexpression of NRF-1, which lead to an increase in Parkin and PINK1 gene and protein expression, while silencing of NRF-1 lead to Parkin and PINK1 downregulation. Moreover, Parkin mutations and consequential downregulation of PGC $-1 \alpha$ causes loss of NRF- 1 and TFAM.

TFAM is crucial for the maintenance of the mitochondrial genome. Its functions include the regulation of mtDNA copy number, initiation of mtDNA transcription, participation in mtDNA replication and compaction of the mitochondrial genome into protein-rich structures termed nucleoids. Upon activation by regulatory proteins, such as NRF-1, TFAM translocates from the cytosol to the nucleoid and regulates mitochondrial transcription (Fig. 1).

The mitochondrial genome is located in the mitochondrial matrix and in close proximity to the electron transport chain (ETC) protein complexes. In the process of producing ATP for the cell, toxic by-products, like reactive oxygen species, are also generated and can harm mtDNA molecules (Mecocci et al., 1993). Moreover, deletions in mtDNA accumulate with normal aging in the SN and occur more frequently in PD patients than in age-matched controls (Bender et al., 2006; Kraytsberg et al., 2006). mtDNA depletion and downregulation of TFAM and mtDNA transcription have also been found in post-mortem tissue of PD patients (Grunewald et al., 2016).

Parkin overexpression increases mtDNA-encoded RNA and proteins, which are transcribed by TFAM. Thus, in 2006, Kuroda and colleagues hypothesized that Parkin is associated with TFAM. Using co-immunoprecipitation in COS-1 cells, results showed that Parkin indeed interacts directly with TFAM. Mobility shift assays showed a shift in mtDNA, when TFAM was present, suggesting that Parkin can associate with mtDNA indirectly via TFAM. These findings were reproduced and confirmed on an endogenous level in SH- 


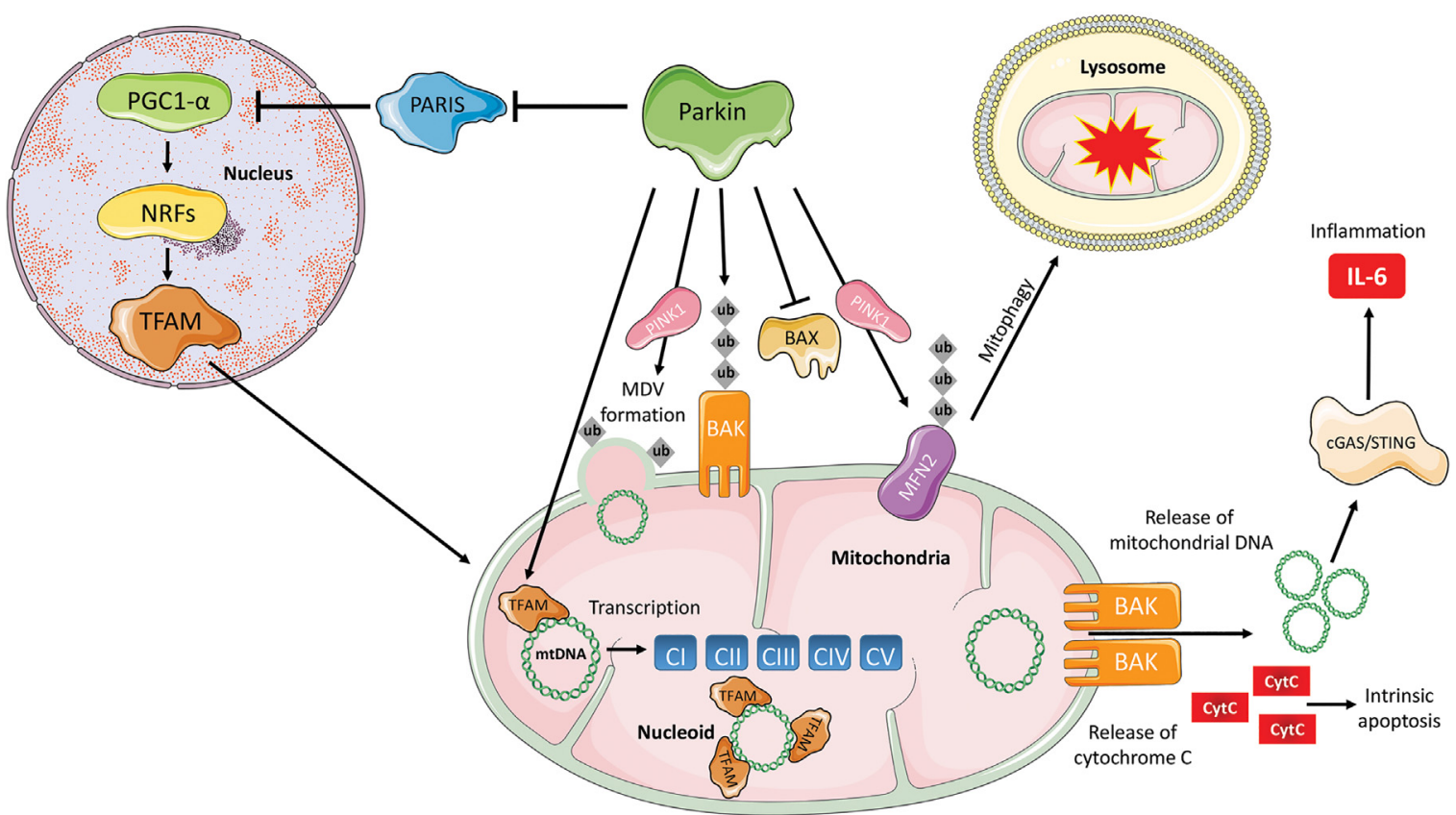

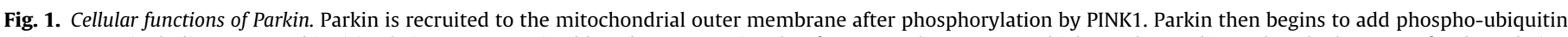

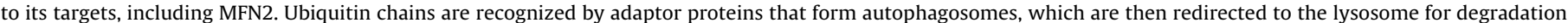

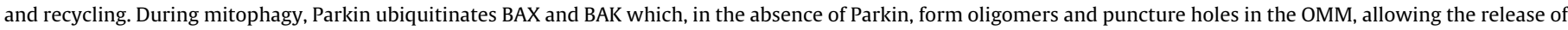

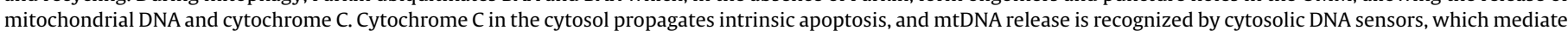

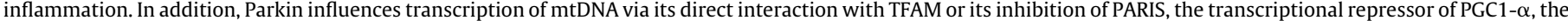

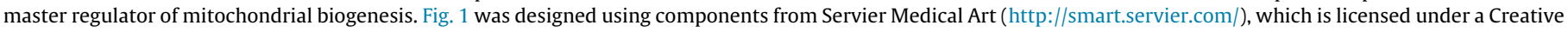
Common Attribution 3.0 Generic License.

A

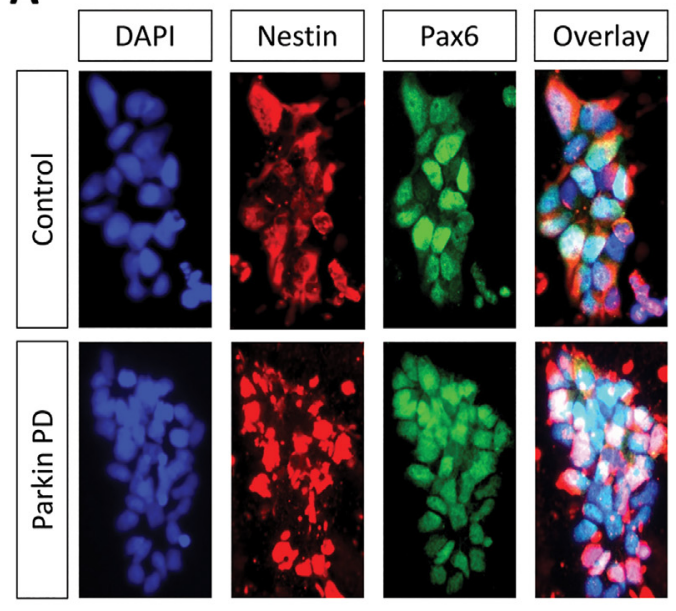

B

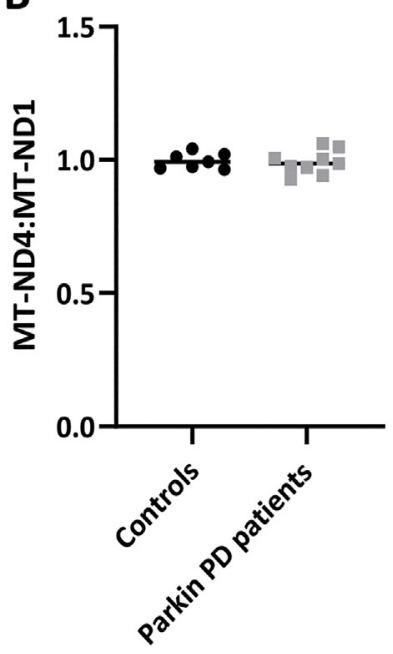

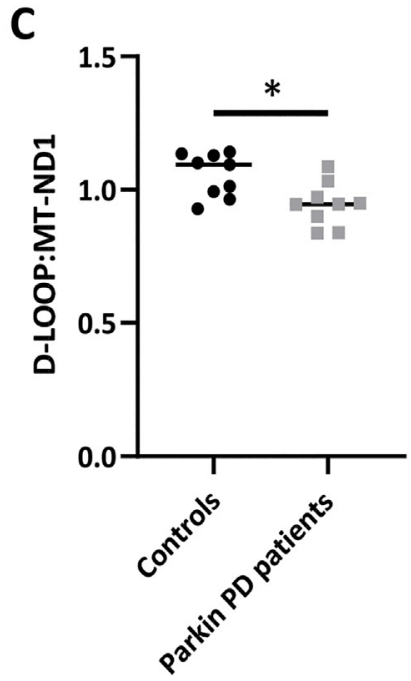

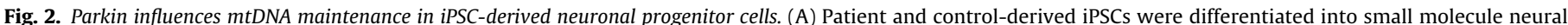

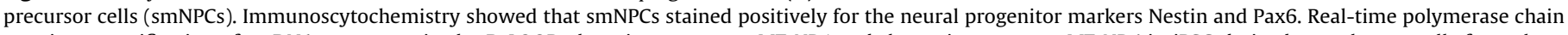

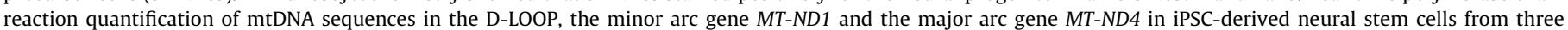

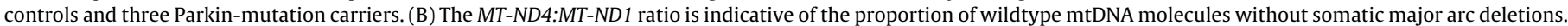

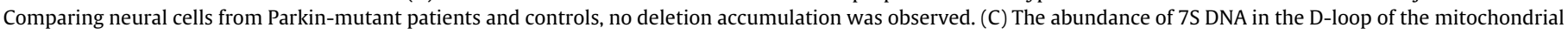

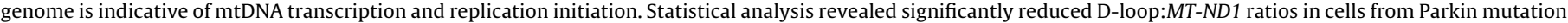
carriers compared to healthy controls. ${ }^{*} \mathrm{p}<0.05$. ND1 $=$ NADH dehydrogenase 1 .

SY5Y cells; ChIP on chip analyses showed that Parkin associates with several mitochondrial genomic sequences including ATPase 6, COXII, ND1, ND2 and D-LOOP. Moreover, Parkin and TFAM coassociate at several of the same mtDNA sequences in post-mitotic neurons and in vivo, and Parkin was shown to protect mitochondrial genome integrity (Rothfuss et al., 2009). By contrast, Parkin's putative role in mtDNA maintenance has not yet been investigated in patient-derived neuronal models with Parkin mutations.
In induced pluripotent stem cell (iPSC)-derived neuronal progenitor cells from Parkin-mutant PD patients and age-matched controls (Fig. 2A), we assessed the abundance of somatic major arc deletions and did not detect differences between the two groups (Fig. 2B). By contrast, when quantifying the presence of 7S DNA, which binds in the D-loop during mtDNA transcription and replication, we observed reduced levels in patient cells (Fig. 2C). This result fur- 
ther implicates impaired mtDNA homeostasis in the pathogenesis of Parkin-linked PD.

While mouse models have unraveled an extraordinary amount of biological mechanisms regarding PD, Parkin-knockout (KO) mice do not display signs of nigrostriatal neurodegeneration or any significant motor phenotypes typical of PD. As variations in the DNA polymerase $\gamma(P O L G)$ gene have been identified as risk factors in PD, Pickrell and colleagues hypothesized that lack of Parkin in mice with increased mutagenic stress may provide a better model to study Parkin-related PD. The mutator-Parkin-KO mouse combines a mutation to the only mtDNA proofreading factor POLG and Parkin $\mathrm{KO}$, subjecting the mice to both mutagenic and loss of Parkin. These mice exhibit PD symptoms and the selective loss of dopaminergic neurons of the SN. In these mice, mtDNA depletion and mutation rate was significantly increased compared to WT mice. Moreover, loss of Parkin expression during mutagenic stress increased the predicted pathogenicity of the incurred mutations (Pickrell et al., 2015).

\section{Inflammation}

In the last decade, an exciting and novel theme has emerged in the pathophysiology of age and aging-related disorders: the role of mitochondria as a trigger for the immune system. Once independent bacteria, mitochondria contain their own DNA located within the mitochondrial matrix. If found outside the matrix, such as the cytosol or extracellular space, mtDNA can be perceived as foreign and potentially toxic. Such molecules released from their natural compartments as a result of cellular stress and/or injury are termed damage-associated molecular patterns (DAMPs) and can initiate a non-infectious inflammatory response, whereby cytosolic and/or extracellular pattern recognition receptors (PRRs) recognize and activate inflammatory pathways to prompt innate immunity (Roh and Sohn, 2018).

TFAM is the major protein comprising nucleoids, and TFAM downregulation has been shown in post mortem idiopathic PD brains and correlates with a loss of 7S DNA-associated mtDNA transcription and depletion (Grunewald et al., 2016). In TFAM-knock down mouse embryonic fibroblasts, loss of TFAM leads to mtDNA escape into the cytoplasm prompting its "foreign" recognition and subsequent activation of the cyclic GMP-AMP synthase-stimulator of interferon genes (cGAS-STING) innate immune response, thereby resulting in proinflammatory cytokine signaling (Fig. 1) (West et al., 2015).

As mitophagy selectively degrades malfunctioning mitochondria, it is plausible that mitophagy may reduce the expulsion of DAMPs from mitochondria. Compared to wild type mice, Parkindeficient mice presented with significantly higher concentrations of IL-6, IFN 31 , IL-12 p70, IL-13, CXCL1, CCL2 and CCL4 in serum, which was dependent on cGas-STING activation. Human serum from biallelic Parkin-mutation carriers also showed significantly higher levels of IL-6, IL-1 $\beta$, and CCL4 compared to healthy controls, suggesting that lack of Parkin results in an increase in cytokines preceding symptomatic neurodegeneration (Sliter et al., 2018). IFN $\beta 1$, a type 1 interferon, is produced upon STING activation. In similar fashion, in our recent biomarker study, serum from Parkin-mutation carriers showed higher levels of cell-free circulating mtDNA and IL-6 levels compared to both idiopathic PD patients and healthy controls. Moreover, IL-6 levels correlated with disease duration in Parkin mutation carriers (Borsche et al., 2020).

\section{Treatment}

The overwhelming majority of Parkin mutation carriers respond favorably to Levodopa treatment (>98 \%). Of those with reported information, $93 \%$ respond well to Levodopa and only a small minority shows a moderate $(4 \%)$ or minimal (3\%) response, respectively (www.mdsgene.org). Although prospective studies are still lacking, it appears that deep brain stimulation is as effective in Parkin mutation carriers as in idiopathic PD patients (de Oliveira et al., 2019; Kim et al., 2014; Rizzone et al., 2019).

More recently, therapeutic approaches are being explored that target specific Parkin mutation-induced (dys-)function including mitophagy. A small molecule activator of mitophagy, either activating Parkin or PINK1 directly or inhibiting Parkin's counterpart, the ubiquitin-specific protease USP30, are in preclinical development (Miller and Muqit, 2019). It has also been argued that Parkin-PD patients would be particularly well-suited candidates for dopaminergic cell replacement therapies based on the frequent confinement of their neurodegeneration to the nigrostriatal pathway and on their overall younger age and rarer occurrence of comorbidities (Kunath et al., 2019). In this context, it is exciting to note that patient-derived midbrain dopaminergic progenitor cells, differentiated in vitro from autologous iPSCs, were successfully implanted in a patient with idiopathic PD. The cells were demonstrated to have the phenotypic properties of SN pars compacta neurons and were implanted into both putamina without the need for immunosuppression. Positron-emission tomography with the use of fluorine-18-L-dihydroxyphenylalanine indicated graft survival which was paralleled by stabilization or even improvement of PD signs and symptoms at 18-24 months after implantation (Schweitzer et al., 2020). It remains to be explored whether implantation of (mutation-corrected) autologous iPSC-derived neurons would be an equally viable therapeutic strategy for Parkin-linked PD.

Finally, the detection of elevated cytokine levels in the serum of patients with Parkin mutations suggests that anti-inflammatory treatments could be considered to modulate the progression of PD in these cases (Borsche et al., 2020).

\section{Acknowledgements}

CK receives funding within the framework of SysMedPD (European Union's Horizon 2020 research and innovation program). CK and AG are funded by the German Research Foundation (FOR2488, GR 3731/5-1). AG and KW are supported by the Luxembourg National Research Fund (ATTRACT career development grant, FNR9631103; INTER grant, FNR11250962).

\section{References}

Alves da Costa, C., Duplan, E., Rouland, L., Checler, F., 2018. The transcription factor function of Parkin: breaking the dogma. Front. Neurosci. 12, 965.

Bender, A., Krishnan, K.J., Morris, C.M., Taylor, G.A., Reeve, A.K., Perry, R.H., Jaros, E., Hersheson, J.S., Betts, J., Klopstock, T., Taylor, R.W., Turnbull, D.M., 2006. High levels of mitochondrial DNA deletions in substantia nigra neurons in aging and Parkinson disease. Nat. Genet. 38, 515-517.

Bernardini, J.P., Brouwer, J.M., Tan, I.K., Sandow, J.J., Huang, S., Stafford, C.A., Bankovacki, A., Riffkin, C.D., Wardak, A.Z., Czabotar, P.E., Lazarou, M., Dewson, G., 2019. Parkin inhibits BAK and BAX apoptotic function by distinct mechanisms during mitophagy. EMBO J., 38.

Borsche, M., König, I.R., Delcambre, S., Petrucci, S., Balck, A., Brüggemann, N., Zimprich, A., Wasner, K., Pereira, S.L., Avenali, M., Deuschle, C., Badanjak, K., Ghelfi, J., Gasser, T., Kasten, M., Rosenstiel, P., Lohmann, K., Brockmann, K., Valente, E.M., Youle, R.J., Grünewald, A., Klein, C., in press. Mitochondrial damage-associated inflammation highlights biomarkers in PRKN/PINK1 parkinsonism. Brain.

de Oliveira, L.M., Barbosa, E.R., Aquino, C.C., Munhoz, R.P., Fasano, A., Cury, R.G., 2019 Deep brain stimulation in patients with mutations in Parkinson's disease-related genes: a systematic review. Mov. Disord. Clin. Pract. 6, 359-368.

Deng, H., Dodson, M.W., Huang, H., Guo, M., 2008. The Parkinson's disease genes pink1 and parkin promote mitochondrial fission and/or inhibit fusion in Drosophila. Proc. Natl. Acad. Sci. U. S. A. 105, 14503-14508.

Dorsey, E.R., Bloem, B.R., 2018. The Parkinson Pandemic-A call to action. JAMA Neurol. 75, 9-10.

Geisler, S., Holmstrom, K.M., Skujat, D., Fiesel, F.C., Rothfuss, O.C., Kahle, P.J., Springer, W., 2010. PINK1/Parkin-mediated mitophagy is dependent on VDAC1 and p62/SQSTM1. Nat. Cell Biol. 12, 119-131. 
Grunewald, A., Kasten, M., Ziegler, A., Klein, C., 2013. Next-generation phenotyping using the parkin example: time to catch up with genetics. JAMA Neurol. 70, 1186-1191.

Grunewald, A., Rygiel, K.A., Hepplewhite, P.D., Morris, C.M., Picard, M., Turnbull, D.M., 2016. Mitochondrial DNA depletion in respiratory chain-deficient parkinson disease neurons. Ann. Neurol. 79, 366-378.

Gugneja, S., Virbasius, C.M., Scarpulla, R.C., 1996. Nuclear respiratory factors 1 and 2 utilize similar glutamine-containing clusters of hydrophobic residues to activate transcription. Mol. Cell. Biol. 16, 5708-5716.

Kane, L.A., Lazarou, M., Fogel, A.I., Li, Y., Yamano, K., Sarraf, S.A., Banerjee, S., Youle, R.J., 2014. PINK1 phosphorylates ubiquitin to activate Parkin E3 ubiquitin ligase activity. J. Cell Biol. 205, 143-153.

Kasten, M., Hartmann, C., Hampf, J., Schaake, S., Westenberger, A., Vollstedt, E.J., Balck, A., Domingo, A., Vulinovic, F., Dulovic, M., Zorn, I., Madoev, H., Zehnle, H., Lembeck, C.M., Schawe, L., Reginold, J., Huang, J., Konig, I.R., Bertram, L., Marras, C., Lohmann, K., Lill, C.M., Klein, C., 2018. Genotype-phenotype relations for the Parkinson's disease genes parkin, PINK1, DJ1: MDSGene systematic review. Mov. Disord. 33, 730-741.

Kim, H.J., Yun, J.Y., Kim, Y.E., Lee, J.Y., Kim, H.J., Kim, J.Y., Park, S.S., Paek, S.H., Jeon, B.S., 2014. Parkin mutation and deep brain stimulation outcome. J. Clin. Neurosci. 21, $107-110$

Kitada, T., Asakawa, S., Hattori, N., Matsumine, H., Yamamura, Y., Minoshima, S., Yokochi, M., Mizuno, Y., Shimizu, N., 1998. Mutations in the parkin gene cause autosomal recessive juvenile parkinsonism. Nature 392, 605-608.

Klein, C., Pramstaller, P.P., Kis, B., Page, C.C., Kann, M., Leung, J., Woodward, H., Castellan, C.C., Scherer, M., Vieregge, P., Breakefield, X.O., Kramer, P.L., Ozelius, L.J., 2000. Parkin deletions in a family with adult-onset, tremor-dominant parkinsonism: expanding the phenotype. Ann. Neurol. 48, 65-71.

Kraytsberg, Y., Kudryavtseva, E., McKee, A.C., Geula, C., Kowall, N.W., Khrapko, K., 2006. Mitochondrial DNA deletions are abundant and cause functional impairment in aged human substantia nigra neurons. Nat. Genet. 38, 518-520.

Kunath, T., Natalwala, A., Chan, C., Chen, Y., Stecher, B., Taylor, M., Khan, S., Muqit, M.M.K., 2019. Are PARKIN patients ideal candidates for dopaminergic cell replacement therapies? Eur. J. Neurosci. 49, 453-462.

Kuroda, Y., Mitsui, T., Kunishige, M., Shono, M., Akaike, M., Azuma, H., Matsumoto, T., 2006. Parkin enhances mitochondrial biogenesis in proliferating cells. Hum. Mol. Genet. 15, 883-895.

Lucking, C.B., Durr, A., Bonifati, V., Vaughan, J., De Michele, G., Gasser, T., Harhangi, B.S., Meco, G., Denefle, P., Wood, N.W., Agid, Y., Brice, A., 2000. French Parkinson's Disease Genetics Study, G., European Consortium on Genetic Susceptibility in Parkinson's, D, Association between early-onset Parkinson's disease and mutations in the parkin gene. N. Engl. J. Med. 342, 1560-1567.

McLelland, G.L., Soubannier, V., Chen, C.X., McBride, H.M., Fon, E.A., 2014. Parkin and PINK1 function in a vesicular trafficking pathway regulating mitochondrial quality control. EMBO J. 33, 282-295.

Mecocci, P., MacGarvey, U., Kaufman, A.E., Koontz, D., Shoffner, J.M., Wallace, D.C., Beal, M.F., 1993. Oxidative damage to mitochondrial DNA shows marked agedependent increases in human brain. Ann. Neurol. 34, 609-616.

Miller, S., Muqit, M.M.K., 2019. Therapeutic approaches to enhance PINK1/Parkin mediated mitophagy for the treatment of Parkinson's disease. Neurosci. Lett. 705, 7-13.

Narendra, D., Tanaka, A., Suen, D.F., Youle, R.J., 2008. Parkin is recruited selectively to impaired mitochondria and promotes their autophagy. J. Cell Biol. 183, 795-803.

Parkinson, J., 2002. An essay on the shaking palsy. 1817. J. Neuropsychiatry Clin. Neurosci. 14, 223-236, discussion 222.
Pickrell, A.M., Huang, C.H., Kennedy, S.R., Ordureau, A., Sideris, D.P., Hoekstra, J.G., Harper, J.W., Youle, R.J., 2015. Endogenous parkin preserves dopaminergic substantia nigral neurons following mitochondrial DNA mutagenic stress. Neuron 87, 371-381.

Rizzone, M.G., Martone, T., Balestrino, R., Lopiano, L., 2019. Genetic background and outcome of Deep Brain Stimulation in Parkinson's disease. Parkinsonism Relat. Disord. 64, 8-19.

Roh, J.S., Sohn, D.H., 2018. Damage-associated molecular patterns in inflammatory diseases. Immune Netw. 18, e27.

Rothfuss, O., Fischer, H., Hasegawa, T., Maisel, M., Leitner, P., Miesel, F., Sharma, M., Bornemann, A., Berg, D., Gasser, T., Patenge, N., 2009. Parkin protects mitochondrial genome integrity and supports mitochondrial DNA repair. Hum. Mol. Genet. 18, 3832-3850.

Sarraf, S.A., Raman, M., Guarani-Pereira, V., Sowa, M.E., Huttlin, E.L., Gygi, S.P., Harper J.W., 2013. Landscape of the PARKIN-dependent ubiquitylome in response to mitochondrial depolarization. Nature 496, 372-376.

Schweitzer, J.S., Song, B., Herrington, T.M., Park, T.Y., Lee, N., Ko, S., Jeon, J., Cha, Y., Kim, K., Li, Q., Henchcliffe, C., Kaplitt, M., Neff, C., Rapalino, O., Seo, H., Lee, I.H., Kim, J., Kim, T., Petsko, G.A., Ritz, J., Cohen, B.M., Kong, S.W., Leblanc, P., Carter B.S., Kim, K.S., 2020. Personalized iPSC-Derived dopamine progenitor cells for Parkinson's disease. N. Engl. J. Med. 382, 1926-1932.

Seirafi, M., Kozlov, G., Gehring, K., 2015. Parkin structure and function. FEBS J. 282, 2076-2088.

Shiba-Fukushima, K., Imai, Y., Yoshida, S., Ishihama, Y., Kanao, T., Sato, S., Hattori, N., 2012. PINK1-mediated phosphorylation of the Parkin ubiquitin-like domain primes mitochondrial translocation of Parkin and regulates mitophagy. Sci. Rep. 2, 1002 .

Shin, J.H., Ko, H.S., Kang, H., Lee, Y., Lee, Y.I., Pletinkova, O., Troconso, J.C., Dawson, V.L., Dawson, T.M., 2011. PARIS (ZNF746) repression of PGC-1alpha contributes to neurodegeneration in Parkinson's disease. Cell 144, 689-702.

Sliter, D.A., Martinez, J., Hao, L., Chen, X., Sun, N., Fischer, T.D., Burman, J.L., Li, Y., Zhang, Z., Narendra, D.P., Cai, H., Borsche, M., Klein, C., Youle, R.J., 2018. Parkin and PINK1 mitigate STING-induced inflammation. Nature 561, 258-262.

Stevens, D.A., Lee, Y., Kang, H.C., Lee, B.D., Lee, Y.I., Bower, A., Jiang, H., Kang, S.U. Andrabi, S.A., Dawson, V.L., Shin, J.H., Dawson, T.M., 2015. Parkin loss leads to PARIS-dependent declines in mitochondrial mass and respiration. Proc. Natl. Acad. Sci. U. S. A. 112, 11696-11701.

Suliman, H.B., Keenan, J.E., Piantadosi, C.A., 2017. Mitochondrial quality-control dysregulation in conditional HO-1(-/-) mice. JCI Insight 2, e89676.

Tan, M.M.X., Malek, N., Lawton, M.A., Hubbard, L., Pittman, A.M., Joseph, T., Hehir, J., Swallow, D.M.A., Grosset, K.A., Marrinan, S.L., Bajaj, N., Barker, R.A., Burn, D.J., Bresner, C., Foltynie, T., Hardy, J., Wood, N., Ben-Shlomo, Y., Grosset, D.G., Williams, N.M., Morris, H.R., 2019. Genetic analysis of Mendelian mutations in a large UK population-based Parkinson's disease study. Brain 142, 2828-2844.

West, A.P., Khoury-Hanold, W., Staron, M., Tal, M.C., Pineda, C.M., Lang, S.M., Bestwick, M., Duguay, B.A., Raimundo, N., MacDuff, D.A., Kaech, S.M., Smiley, J.R., Means, R.E., Iwasaki, A., Shadel, G.S., 2015. Mitochondrial DNA stress primes the antiviral innate immune response. Nature 520, 553-557.

Zheng, L., Bernard-Marissal, N., Moullan, N., D’Amico, D., Auwerx, J., Moore, D.J. Knott, G., Aebischer, P., Schneider, B.L., 2017. Parkin functionally interacts with PGC-1alpha to preserve mitochondria and protect dopaminergic neurons. Hum. Mol. Genet. 26, 582-598. 